\title{
Vertebral multiple myeloma with pathological fracture: the most common etiology for emergency spine surgery in patients with no cancer diagnosis on admission
}

\author{
Joshua D. Burks, MD, Turki Elarjani, MD, Aria M. Jamshidi, MD, Vaidya Govindarajan, BS, and \\ Allan D. Levi, MD, PhD \\ Department of Neurological Surgery, University of Miami Miller School of Medicine, Miami, Florida
}

\begin{abstract}
OBJECTIVE Vertebral compression fractures are common in multiple myeloma (MM). Modern treatment paradigms place emphasis on treatment with radiation, with surgery reserved for cases involving frank instability or severe neural compression. However, experience at the authors' institution has led them to suspect a more prominent role for surgical intervention in some settings. The authors undertook the present study to better understand the incidence of MM in undiagnosed patients who require urgent surgery for pathological vertebral fracture.
\end{abstract}

METHODS The authors reviewed a prospectively collected database of all patients who underwent surgery with the senior author at their main hospital between June 1, 1998, and June 30, 2020. Patients admitted from the emergency room or after transfer from another hospital who then underwent surgery for pathological fracture during the same admission were included in the final analysis. Patients scheduled for elective surgery and those with previous cancer diagnoses were excluded.

RESULTS Forty-three patients were identified as having undergone urgent surgical decompression and/or stabilization for pathological fracture. Histopathology confirmed diagnosis of MM in $22(51 \%)$ patients, lung metastasis in $5(12 \%)$ patients, and breast metastasis in $4(9 \%)$ patients. Twelve (28\%) patients were diagnosed with other types of metastatic carcinoma or undifferentiated disease. Sixteen of $29(55 \%)$ men and 6 of $14(42 \%)$ women were diagnosed with MM $(p=$ 0.02). Seventeen of $34(50 \%)$ patients who underwent surgery for neurological deficit, 5 of $6(83 \%)$ patients who underwent surgery for spinal instability, and $0(0 \%)$ patients who underwent surgery for pain with impending spinal cord injury were diagnosed with $\mathrm{MM}(\mathrm{p}=0.12)$.

CONCLUSIONS A majority of patients presenting to the authors' hospital with no history of malignancy who required urgent surgery for pathological compression fracture were found to have MM or plasmacytoma. This disease process may affect a significant portion of patients requiring decompressive or stabilizing surgery for compression fracture in academic medical centers.

https://thejns.org/doi/abs/10.3171/2021.2.FOCUS201038

KEYWORDS multiple myeloma; neurosurgery; pathological; fracture; incidence; operative

$\mathrm{M}$ ULTIPLE myeloma (MM) is a disease caused by the diffuse, uncontrolled proliferation of plasma cells. Recent estimates indicate that nearly 140,000 new cases are diagnosed each year worldwide. ${ }^{1}$ Bone disease occurs in $80 \%$ of patients, with bone pain as the first symptom in $70 \%$ of patients. ${ }^{2}$ Vertebral compression fractures occur in 55\%-70\%, ${ }^{3}$ sometimes resulting in significant deformity with or without neural compression. Solitary plasmacytoma is a precursor to $\mathrm{MM},{ }^{4}$ characterized by a tumor mass that results from the focal proliferation of plasma cells without systemic involvement of the bone marrow. Spinal involvement is thought to be promi- nent in both entities, given the fact that vertebral bodies contain a high amount of hematopoietic bone marrow compared to other parts of the body. ${ }^{5}$

The role surgery plays in treating pathological fractures caused by MM and plasmacytoma has long been a topic of debate, because procedural intervention attains rapid relief of symptoms but with high rates of morbidity and mortality ${ }^{6,7}$ Patients with MM are at higher risk for thromboembolic complications and have a suppressed immune system, resulting in higher rates of infection. ${ }^{8,9}$ Some authors have described favorable outcomes in selected patients with surgical stabilization. Particularly in patients with

ABBREVIATIONS ER = emergency room; ESCC = epidural spinal cord compression; $M M$ = multiple myeloma ACCOMPANYING EDITORIAL DOI: 10.3171/2021.2.FOCUS21108.

SUBMITTED December 4, 2020. ACCEPTED February 16, 2021.

INCLUDE WHEN CITING DOI: 10.3171/2021.2.FOCUS201038. 
preoperative deficits, surgery has been effective in improving functioning and restoring structural stability. ${ }^{10-14}$ Other authors have suggested cement augmentation as a less invasive alternative to improve structural deformity and alleviate pain in several series. ${ }^{15-19}$ However, other studies have demonstrated favorable outcomes in patients treated with radiation alone, even in the presence of neural compression or evidence of radiographic instability. ${ }^{20,21}$

Current international guidelines recommend primary treatment with radiation unless there is evidence of frank instability or rapid neurological deterioration necessitating surgery first. ${ }^{22}$ Indeed, neurosurgical trainees are taught that MM is a disease treated primarily with radiotherapy unless there are extenuating circumstances. ${ }^{23}$ Coupled with earlier disease detection following the advent of MRI, ${ }^{24}$ these factors have contributed to a consensus within the neurosurgical community that MM is generally not a surgically treated disease.

However, experience at our institution has led us to suspect a more prominent role for surgery in MM in some practice settings. In particular, patients with no history of malignancy frequently present to our tertiary referral center at a large public hospital with pathological fracture requiring urgent decompression and stabilization. Many are later diagnosed with MM or plasmacytoma after surgery, having been subjected to a rapid, preoperative metastatic workup that failed to yield a diagnosis. We undertook this study to quantify the incidence of MM in undiagnosed patients who required urgent surgery for pathological vertebral fracture.

\section{Methods}

This study was conducted after approval by the institutional review board. We reviewed a prospectively collected database of all patients who underwent surgery with the senior author (A.D.L.) at a single hospital between June 1, 1998, and June 30, 2020. Lesion characteristics, demographic information, and surgical indications were recorded for patients with pathological fracture who were treated surgically. Surgical indications were delineated as neurological deficit, spinal instability, or refractory pain with impending spinal cord injury. Only patients admitted from the emergency room (ER) or after transfer from another hospital who then underwent surgery during the same admission were included in the final analysis. Additionally, patients were only included if there was no previous cancer diagnosis prior to arrival in the ER, or if the patient had been treated for cancer more than 10 years prior with no other evidence of recurrence. Patients who were scheduled for surgery on an elective basis also were not included.

We defined pathological fracture as a fracture occurring either spontaneously or after minor trauma, secondary to malignancy arising from the vertebral body and resulting in bony deformity. In all cases, lesions were imaged with CT and contrasted MRI and classified as pathological fracture preoperatively by the senior author and an attending neuroradiologist. All treated fractures had evidence of epidural spinal cord compression (ESCC) or nerve root compression (Bilsky grade 2-3). ${ }^{25}$ Patients with intradural disease, with disease exhibiting extensive infil-
TABLE 1. Characteristics in 43 patients with pathological fractures

\begin{tabular}{cc}
\hline Characteristic & No. $(\%)$ \\
\hline Sex & $16(37)$ \\
\hline Female & $27(63)$ \\
\hline Male & $9(21)$ \\
\hline Race & $13(30)$ \\
\hline Black & $19(44)$ \\
\hline Hispanic & $2(5)$ \\
\hline Non-Hispanic White & \\
\hline Unknown & $4(9)$ \\
\hline Pathology & $5(12)$ \\
\hline Breast mets & $12(28)$ \\
\hline Lung mets & $22(51)$ \\
\hline Other mets & $30(70)$ \\
\hline MM/plasmacytoma & \\
\hline Neurological deficit & $9(21)$ \\
\hline Involved spinal level & $25(58)$ \\
\hline Cervical & $9(21)$ \\
\hline Thoracic &
\end{tabular}

Mets $=$ metastasis .

tration of the paraspinal musculature and/or viscera consistent with connective tissue malignancy, or with characteristics strongly suggestive of primary bone tumors were excluded.

\section{Statistical Analysis}

Continuous variables are reported as means with standard deviation for normally distributed data, and medians with interquartile range for nonnormally distributed data. Categorical variables are reported by frequency. Betweengroup comparisons were performed with Pearson chisquare and 1-way ANOVA tests. Fisher's exact test was used when greater than $80 \%$ of values were less than 5 . All statistical analysis was performed using IBM SPSS (version 24; IBM Corp.).

\section{Results}

A total of 43 patients were identified as having undergone urgent surgical decompression and/or stabilization for pathological fracture with the senior author. Histopathology confirmed diagnosis of MM in $22(51 \%)$ patients, lung metastasis in $5(12 \%)$ patients, and breast metastasis in $4(9 \%)$ patients. The remaining $12(28 \%)$ patients were diagnosed with other types of metastatic carcinoma or undifferentiated disease. The thoracic spine was primarily involved in $25(58 \%)$ cases, whereas the cervical and lumbar spine were involved in $9(21 \%)$ cases each. Other characteristics of included patients are given in Table 1.

Sixteen of $29(55 \%)$ men and 6 of $14(42 \%)$ women were diagnosed with MM (p=0.02). Seventeen of $34(50 \%)$ patients who underwent surgery for neurological deficit, 5 of $6(83 \%)$ patients who underwent surgery for spinal insta- 
TABLE 2. Comparison of histological diagnosis in patients with pathological fracture undergoing surgery during the same admission

\begin{tabular}{|c|c|c|c|c|c|}
\hline \multirow[b]{2}{*}{ Characteristic } & \multicolumn{4}{|c|}{ No. w/ Pathology (\%) } & \multirow[b]{2}{*}{$p$ Value } \\
\hline & Breast Mets & Lung Mets & Other Mets & MM/Plasmacytoma & \\
\hline \multicolumn{6}{|l|}{ Sex } \\
\hline Female & $4(29)$ & $0(0)$ & $4(29)$ & $6(42)$ & \multirow{2}{*}{$0.02^{*}$} \\
\hline Male & $0(0)$ & $5(17)$ & $8(28)$ & $16(55)$ & \\
\hline \multicolumn{6}{|l|}{ Race } \\
\hline Black & $1(11)$ & $2(23)$ & $3(33)$ & $3(33)$ & \multirow{3}{*}{$0.39^{*}$} \\
\hline Hispanic & $0(0)$ & $2(15)$ & $2(15)$ & $9(70)$ & \\
\hline Non-Hispanic White & $3(16)$ & $1(5)$ & $6(32)$ & $9(47)$ & \\
\hline \multicolumn{6}{|l|}{ Surgical indications } \\
\hline Neurological deficit & $2(6)$ & $5(15)$ & $10(29)$ & $17(50)$ & \multirow{3}{*}{0.12} \\
\hline Spinal instability & $1(17)$ & $0(0)$ & $0(0)$ & $5(83)$ & \\
\hline Pain & $1(33)$ & $0(0)$ & $2(67)$ & $0(0)$ & \\
\hline \multicolumn{6}{|l|}{ Spinal level } \\
\hline Cervical & $0(0)$ & $1(11)$ & $2(22)$ & $6(67)$ & \multirow{3}{*}{$0.89^{*}$} \\
\hline Thoracic & $4(16)$ & $3(12)$ & $7(28)$ & $11(44)$ & \\
\hline Lumbar & $0(0)$ & $1(11)$ & $3(33)$ & $5(56)$ & \\
\hline Age in yrs, mean \pm SD & $54 \pm 13$ & $61 \pm 5$ & $59 \pm 11$ & $58 \pm 12$ & 0.83 \\
\hline
\end{tabular}

* Statistically significant according to Fisher's exact test.

bility, and $0(0 \%)$ patients who underwent surgery for pain with impending spinal cord injury were diagnosed with MM ( $\mathrm{p}=0.12)$. Patients diagnosed with MM had a mean age of 58 years, and patients diagnosed with breast, lung, and other/undifferentiated metastasis had a mean age of 54,61 , and 59 years, respectively $(\mathrm{p}=0.83)$. Comparison of presenting features by histopathological diagnosis are given in Table 2. A sample case is shown in Fig. 1.

\section{Discussion}

Most cases of MM will have spinal involvement, and surgery is considered in instances in which patients present with neural compression or significant instability. However, MM is generally not regarded as a surgical disease because management strategies include a combination of autologous stem-cell transplant, radiation, and chemotherapy. ${ }^{26}$ Nevertheless, we have suspected that in some practice settings MM is more often treated first with surgery.

In this study we reviewed the incidence of MM in patients with no history of malignancy who presented with pathological fracture that required urgent surgery at our hospital. We found that a majority of patients were diagnosed with MM (51\%). There was a tendency for these patients to be male $(73 \%, \mathrm{p}=0.02)$, and most patients
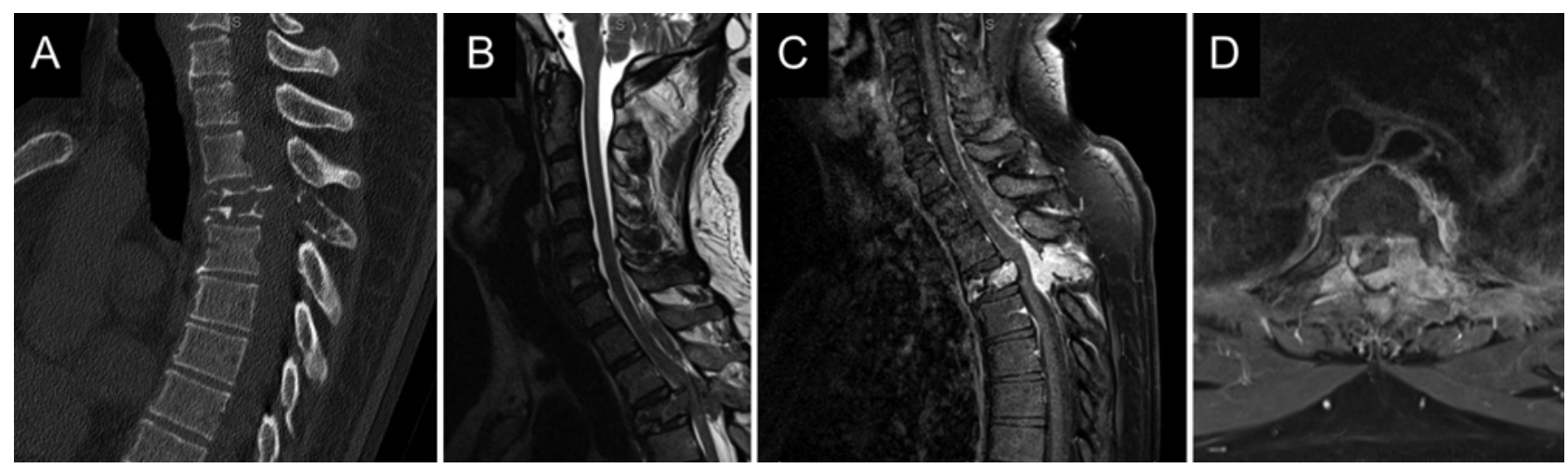

FIG. 1. MM may be the most common cause of pathological fracture requiring urgent surgery in some practice settings. A characteristic case presentation is shown. In this instance, a 56-year-old man with 1 month of back pain developed acute-onset paraplegia. CT scans revealed pathological fracture involving the T3 vertebral body with significant retropulsion, shown in the sagittal plane (A), as well as destruction of the posterior bony elements. Sagittal T2-weighted MRI demonstrates severe cord compression with increased cord signal adjacent to the fracture (B), and epidural disease is noted on T1-weighted imaging with contrast in the sagittal (C) and axial (D) planes, extending from T1 to T4, producing compression of the thecal sac and spinal cord. 
who underwent surgery for MM had a neurological deficit $(77 \%, \mathrm{p}=0.12)$. For all pathology types, fractures of the thoracic spine were most common, accounting for $58 \%$ of all fractures seen.

Widely accepted treatment algorithms suggest treatment of ESCC with radiation therapy in cases in which tumors are known to be radiosensitive. ${ }^{22}$ However, a significant number of patients with MM still ultimately undergo surgery. Earlier studies suggest that as many as 20\%-40\% of patients with MM or plasmacytoma undergo some form of spine operation..$^{13,27,28}$ The prevalence of surgery despite the proven efficacy of radiation is likely to be attributable in part to the effectiveness of surgery in alleviating neurological symptoms, including paraparesis. Among patients with spinal cord injury secondary to metastatic disease, lung metastasis and $\mathrm{MM} /$ plasmacytoma are the most common pathologies. ${ }^{29}$

Other surgical guidelines recognize a need for surgery in some instances of ESCC secondary to metastatic disease. A 2016 review by Laufer and colleagues supported surgery for progressive neurological deficits in the setting of ESCC secondary to metastatic disease. They recommend careful consideration of duration and severity of symptoms in evaluating patients for surgery, as well as oncological burden-noting, however, that decompression should not be delayed to establish a diagnosis. ${ }^{30}$ Other characteristics to consider include spinal level involved, mechanical pain, lytic appearance, subluxation, severity of vertebral body collapse, and involvement of posterior spinal elements. ${ }^{31}$ Based on our results, patients with undiagnosed MM may be more likely to possess features associated with ESCC that require surgery.

In contrast to patients presenting to the clinic for evaluation, often following referral by an oncologist with an established diagnosis, those presenting to the ER at a public hospital often face more barriers to care. Earlier studies have found that among patients seeking care at the ER in urban public hospitals, more than $60 \%$ reported no source of regular care and $48 \%$ reported waiting to seek care. ${ }^{32}$ ER use in public hospitals has also been shown to be the primary source of ambulatory care for patients treated there. ${ }^{33}$ Consequently, presentation and appropriate management may vary widely in comparison to a comprehensive cancer center where patients would be expected to have earlier diagnosis and closer follow-up. In this sense, the patient base is an important consideration when delineating the role for surgery in MM.

Understanding that MM is the most common pathology in cancer-naive patients presenting to the ER with vertebral instability and/or neurological deficit will help the spine surgeon in his or her acute management including diagnostic (e.g., serum electrophoresis) ${ }^{34}$ and treatment decisions.

\section{Limitations}

Our interpretations are offered cautiously given the limitations inherent to prospectively collected patient data that are retrospectively reviewed. Additionally, the characteristics of our patient cohort are probably not applicable to some practice settings such as hospitals specializing in cancer treatment that have a specific referral pattern of patients with a history of cancer.

\section{Conclusions}

A majority of patients presenting to our hospital with no history of malignancy who required urgent surgery for pathological compression fracture were found to have MM or plasmacytoma. This disease process may affect a significant portion of patients requiring decompressive or stabilizing surgery for compression fracture in academic medical centers.

\section{References}

1. Cowan AJ, Allen C, Barac A, et al. Global burden of multiple myeloma: a systematic analysis for the global burden of disease study 2016. JAMA Oncol. 2018;4(9):1221-1227.

2. Kyle RA, Gertz MA, Witzig TE, et al. Review of 1027 patients with newly diagnosed multiple myeloma. Mayo Clin Proc. 2003;78(1):21-33.

3. Lecouvet FE, Vande Berg BC, Maldague BE, et al. Vertebral compression fractures in multiple myeloma. Part I. Distribution and appearance at MR imaging. Radiology. 1997;204(1): 195-199.

4. Soutar R, Lucraft H, Jackson G, et al. Guidelines on the diagnosis and management of solitary plasmacytoma of bone and solitary extramedullary plasmacytoma. Clin Oncol ( $R$ Coll Radiol). 2004;16(6):405-413.

5. Tosi P. Diagnosis and treatment of bone disease in multiple myeloma: spotlight on spinal involvement. Scientifica (Cairo). 2013;2013:104546.

6. Patel MS, Ghasem A, Greif DN, et al. Evaluating treatment strategies for spinal lesions in multiple myeloma: a review of the literature. Int J Spine Surg. 2018;12(5):571-581.

7. Kyriakou C, Molloy S, Vrionis F, et al. The role of cement augmentation with percutaneous vertebroplasty and balloon kyphoplasty for the treatment of vertebral compression fractures in multiple myeloma: a consensus statement from the International Myeloma Working Group (IMWG). Blood Cancer J. 2019;9(3):27.

8. Fotiou D, Gavriatopoulou M, Terpos E. Multiple myeloma and thrombosis: prophylaxis and risk prediction tools. Cancers (Basel). 2020;12(1):E191.

9. Jacobson DR, Zolla-Pazner S. Immunosuppression and infection in multiple myeloma. Semin Oncol. 1986;13(3):282-290.

10. Dürr HR, Wegener B, Krödel A, et al. Multiple myeloma: surgery of the spine: retrospective analysis of 27 patients. Spine (Phila Pa 1976). 2002;27(3):320-326.

11. Quidet M, Zairi F, Boyle E, et al. Evaluation of the relevance of surgery in patients with multiple myeloma harboring symptomatic spinal involvement: a retrospective case series. World Neurosurg. 2018;114:e356-e365.

12. Zadnik PL, Goodwin CR, Karami KJ, et al. Outcomes following surgical intervention for impending and gross instability caused by multiple myeloma in the spinal column. $J$ Neurosurg Spine. 2015;22(3):301-309.

13. Zhang J, Zhong Y. Clinical analysis of 36 multiple myeloma patients with extramedullary plasmacytoma invasion of the spinal canal. Hematol Oncol. 2015;33(2):75-79.

14. Kehrer M, Koob S, Kehrer A, et al. Multiple myeloma - current standards in surgical treatment. Z Orthop Unfall. 2019; 157(2):164-172.

15. Anselmetti GC, Manca A, Montemurro F, et al. Percutaneous vertebroplasty in multiple myeloma: prospective long-term follow-up in 106 consecutive patients. Cardiovasc Intervent Radiol. 2012;35(1):139-145.

16. Lane JM, Hong R, Koob J, et al. Kyphoplasty enhances function and structural alignment in multiple myeloma. Clin $\mathrm{Or}$ thop Relat Res. 2004;(426):49-53.

17. Dudeney S, Lieberman IH, Reinhardt MK, Hussein M. Ky- 
phoplasty in the treatment of osteolytic vertebral compression fractures as a result of multiple myeloma. J Clin Oncol. 2002;20(9):2382-2387.

18. Huber FX, McArthur N, Tanner M, et al. Kyphoplasty for patients with multiple myeloma is a safe surgical procedure: results from a large patient cohort. Clin Lymphoma Myeloma. 2009;9(5):375-380.

19. Mendoza TR, Koyyalagunta D, Burton AW, et al. Changes in pain and other symptoms in patients with painful multiple myeloma-related vertebral fracture treated with kyphoplasty or vertebroplasty. J Pain. 2012;13(6):564-570.

20. Rao G, Ha CS, Chakrabarti I, et al. Multiple myeloma of the cervical spine: treatment strategies for pain and spinal instability. J Neurosurg Spine. 2006;5(2):140-145.

21. Maranzano E, Latini P. Effectiveness of radiation therapy without surgery in metastatic spinal cord compression: final results from a prospective trial. Int J Radiat Oncol Biol Phys. 1995;32(4):959-967.

22. Spratt DE, Beeler WH, de Moraes FY, et al. An integrated multidisciplinary algorithm for the management of spinal metastases: an International Spine Oncology Consortium report. Lancet Oncol. 2017;18(12):e720-e730.

23. Citow JS, Macdonald RL, Refai D. Comprehensive Neurosurgery Board Review. Thieme; 2011.

24. Ludwig H, Frühwald F, Tscholakoff D, et al. Magnetic resonance imaging of the spine in multiple myeloma. Lancet. 1987;2(8555):364-366.

25. Bilsky MH, Laufer I, Fourney DR, et al. Reliability analysis of the epidural spinal cord compression scale. J Neurosurg Spine. 2010;13(3):324-328.

26. Mikhael J, Ismaila N, Cheung MC, et al. Treatment of multiple myeloma: ASCO and CCO Joint Clinical Practice Guideline. J Clin Oncol. 2019;37(14):1228-1263.

27. Donnarumma P, Tarantino R, Rullo M, et al. Surgery for vertebral involvement in multiple myeloma. J Neurosurg Sci. 2018;62(1):10-15.

28. Wang Y, Li H, Liu C, et al. Solitary plasmacytoma of bone of the spine: results from Surveillance, Epidemiology, and End Results (SEER) registry. Spine (Phila Pa 1976). 2019;44(2): E117-E125.
29. Ge L, Arul K, Mesfin A. Spinal cord injury from spinal tumors: prevalence, management, and outcomes. World Neurosurg. 2019;122:e1551-e1556.

30. Laufer I, Zuckerman SL, Bird JE, et al. Predicting neurologic recovery after surgery in patients with deficits secondary to MESCC: Systematic review. Spine (Phila Pa 1976). 2016; 41(suppl 20):S224-S230.

31. Fisher CG, DiPaola CP, Ryken TC, et al. A novel classification system for spinal instability in neoplastic disease: an evidence-based approach and expert consensus from the Spine Oncology Study Group. Spine (Phila Pa 1976). 2010; 35(22):E1221-E1229.

32. Rask KJ, Williams MV, Parker RM, McNagny SE. Obstacles predicting lack of a regular provider and delays in seeking care for patients at an urban public hospital. JAMA. 1994; 271(24):1931-1933.

33. Rask KJ, Williams MV, McNagny SE, et al. Ambulatory health care use by patients in a public hospital emergency department. J Gen Intern Med. 1998;13(9):614-620.

34. Morrison T, Booth RA, Hauff K, et al. Laboratory assessment of multiple myeloma. Adv Clin Chem. 2019;89:1-58.

\section{Disclosures}

Dr. Levi reports receiving a teaching honorarium from the AANS, grant support from the Department of Defense, and grant support from the NIH.

\section{Author Contributions}

Conception and design: Levi, Jamshidi. Acquisition of data: Burks, Elarjani, Govindarajan. Analysis and interpretation of data: Burks. Drafting the article: Burks. Critically revising the article: all authors. Reviewed submitted version of manuscript: Levi, Burks. Statistical analysis: Burks, Elarjani. Study supervision: Levi.

\section{Correspondence}

Allan D. Levi: University of Miami Miller School of Medicine, Miami,FL.alevi@med.miami.edu. 